\title{
A Model Mechanism-Based Explanation of an In Vitro-In Vivo Disconnect for Improving Extrapolation and Translation
}

\author{
Andrew K. Smith, Yanli Xu, Glen E. P. Ropella, and C. Anthony Hunt \\ Bioengineering and Therapeutic Sciences, University of California, San Francisco, California (A.K.S., Y.X., C.A.H.); and Tempus \\ Dictum, Inc., Milwaukie, Oregon (G.E.P.R.)
}

Received September 2, 2017; accepted February 5, 2018

\begin{abstract}
An improved understanding of in vivo-to-in vitro hepatocyte changes is crucial to interpreting in vitro data correctly and further improving hepatocyte-based in vitro-to-in vivo extrapolations to human targets. We demonstrate using virtual experiments as a means of helping to untangle plausible causes of inaccurate extrapolations. We start with virtual mice that use biomimetic software livers. Previously, using these mice, we discovered model mechanisms that enabled achieving quantitative validation targets while also providing plausible causal explanations for temporal characteristics of acetaminophen hepatotoxicity. We isolated virtual hepatocytes, created a virtual culture, and then conducted doseresponse experiments in both culture and mice. We expected to see differences between the two dose-response curves but were somewhat surprised that they crossed because it evidenced that simulated acetaminophen metabolism and toxicity are different for
\end{abstract}

virtual culture and mouse contexts even though individual hepatocyte mechanisms were unchanged. Differences in dose-response curves provide a virtual example of an in vivo-to-in vitro disconnect. We use detailed results of experiments to explain this disconnect. Individual hepatocytes contribute differently to system-level phenomena. In liver, hepatocytes are exposed to acetaminophen sequentially. Relative production of the reactive acetaminophen metabolite is largest (smallest) in pericentral (periportal) hepatocytes. Because that sequential exposure is absent in culture, hepatocytes from different lobular locations do not respond the same. A virtual culture-to-mouse translation can stand as a scientifically challengeable hypothesis explaining an in vivo-to-in vitro disconnect. It provides a framework to develop more reliable interpretations of in vitro observations, which then may be used to improve extrapolations.

\section{Introduction}

Results of quantitative hepatocyte-based in vitro-to-in vivo extrapolations continue to improve (Poulin, 2016; Tetsuka et al., 2017). In vitro strategies for liver toxicity testing have experienced concurrent advances (Soldatow et al., 2013) aided by increasing knowledge about factors that limit accuracy (LeCluyse et al., 2012; Fraczek et al., 2013; Godoy et al., 2013; Vellonen et al., 2014). Nevertheless, even for straightforward predictions of hepatic clearance from in vitro intrinsic clearance values, published results are typically more than $40 \%$ outside of in vivo values (Bowman and Benet, 2016), and some are 2-fold or more.

Different factors can contribute to inaccurate extrapolations. The following are five examples: 1) variability among livers; 2) loss of lobular architecture and environment; 3) periportal (PP)-to-pericentral (PC) hepatocyte differences; 4) variation in relative numbers of $P P$, midzonal (MZ), and PC hepatocytes in cultured populations; and 5) up- and downregulation of genes during and after isolation coupled with variation in hepatocyte health caused by isolation and/or

The research was funded by the UCSF BioSystems group. https://doi.org/10.1124/jpet.117.245019. culture protocol differences. The prospect of being able to limit or control such changes is motivating interest in threedimensional hepatocyte cultures and microfluidic in vitro systems, with the expectation that they can become reliably more predictive of in vivo and human hepatic phenotypes.

An improved understanding of in vivo-to-in vitro changes is crucial to interpreting in vitro data correctly. We conjecture that insights gained from virtual experiments can help untangle and identify causes of inaccurate extrapolations to in vivo and human targets, particularly contributions from the aforementioned second, third, and fourth factors. The resulting new knowledge may then be used to improve in vitro-to-in vivo extrapolation methods. Figure 1 illustrates the key ideas. There are four essential requisites. 1) One can create many concrete, mechanism-based, individualizable virtual hepatocytes that are demonstrably analogous to actual hepatocytes in particular ways. 2) The three-dimensional organization of virtual hepatocytes can be made strongly analogous to that in rodent and human liver. 3) During execution, we achieve specific qualitative and quantitative validation targets for virtual counterparts of xenobiotics by changing the configurations (parameterizations) of virtual hepatic mechanisms. 4) It is straightforward to isolate all virtual hepatocytes from the simulated liver, without altering intrahepatocyte

ABBREVIATIONS: aHPC, hepatocyte analog; APAP, acetaminophen; CV, central vein; D-R, dose-response; 2D, two-dimensional; GSH, glutathione; GNZ, glutathione and $N$-acetyl- $p$-benzoquinone imine zonation; mitoD, mitochondrial damage; MNZ, mitochondrial damage repair and $N$-acetyl-p-benzoquinone imine zonation; MZ, midzonal; NAPQI, $N$-acetyl-p-benzoquinone imine; NZ, $N$-acetyl- $p$-benzoquinone imine zonation; PC, pericentral; PP, periportal; PV, portal vein; SS, sinusoidal segment. 
A

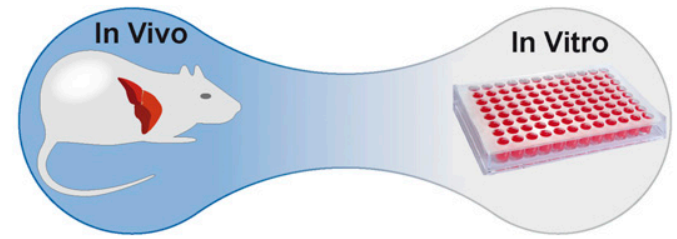

B

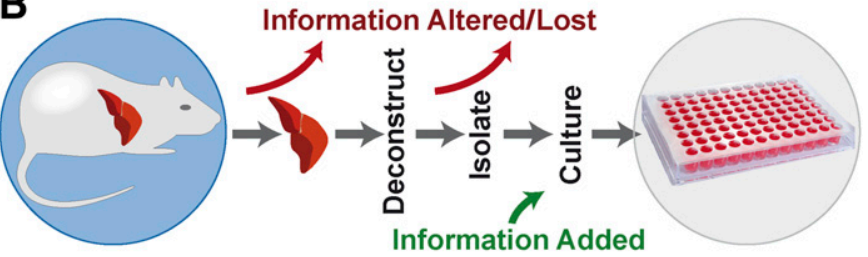

C

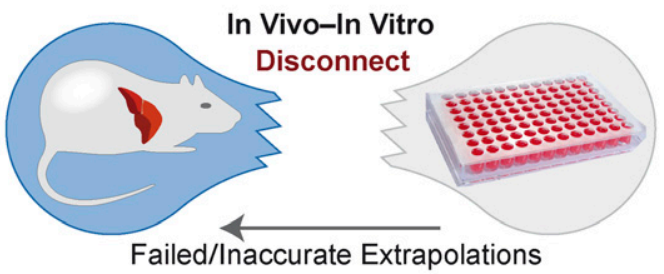

D

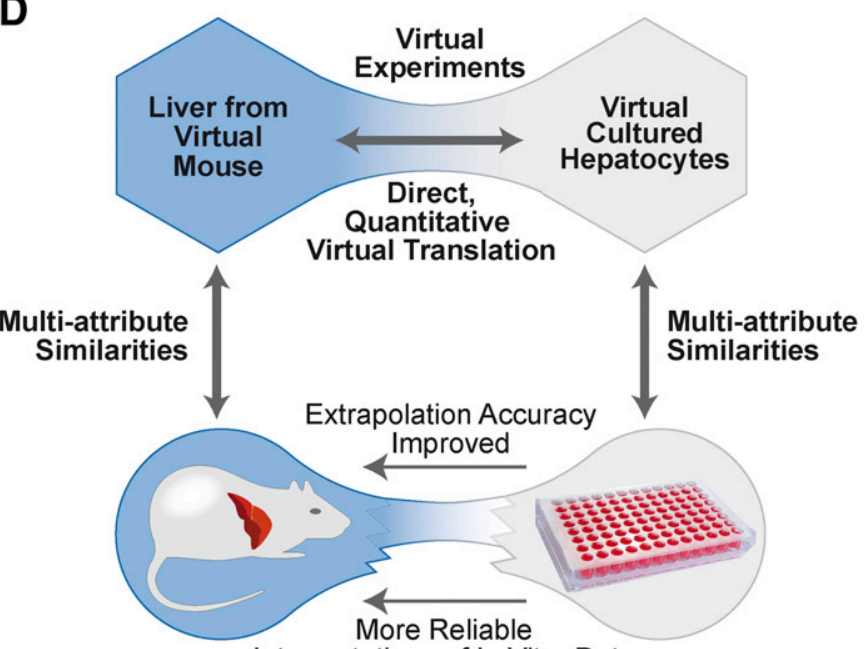

Fig. 1. An approach to improve in vitro-to-in vivo extrapolations: the focus is mechanism-based explanations for differences in phenomena between two model systems. (A) An illustration of an ideal relationship: all phenotypic differences between in vivo and in vitro are understood; therefore, predictions and translations are knowledge based. (B) Some of the knowledge and information required to explain hepatocyte attributes (e.g., metabolic clearance) in vivo is lost and/or added during hepatocyte isolation and culture. (C) The processes illustrated in (B) alter hepatocyte phenotype, creating an in vivo-in vitro disconnect. An extrapolation or prediction based on an altered phenotype is inaccurate or fails to meet requirements. (D) Arrows on each side indicate multiattribute similarities: each virtual system independently builds credibility through cumulative achievement of qualitative and quantitative referent system validation targets. (Top) Differences in phenomena measured during virtual Hepatocyte Culture experiments and corresponding measurements of phenomena recorded during virtual Mouse experiments are entirely explainable: there is no in vivo-in vitro disconnect. (Bottom) Mechanism differences enabling the virtual translation in the top illustration can stand as a concrete, scientifically challengeable hypothesis explaining the disconnect illustrated in (C); that Model Mechanism-based theory provides the framework to develop more reliable interpretations of in vitro observations, which then may be used to improve extrapolations. mechanisms, and then to reorganize them to simulate a twodimensional (2D) hepatocyte culture to study responses to virtual counterparts of xenobiotics. We recently reported using experiments on virtual mice to discover model mechanisms that provide plausible causal explanations for major temporal characteristics of acetaminophen (APAP) hepatotoxicity in mice (Smith et al., 2016). These virtual mice met the first three requisites; they used a software liver analog that is biomimetic across relevant anatomic, hepatic zonation, and cell biology attributes. Quasi-autonomous virtual hepatocytes populate virtual liver lobules. Each virtual hepatocyte uses the local values of PP-to-PC gradients to configure its mechanisms for reactive metabolite formation, glutathione (GSH) depletion, accumulation of mitochondrial damage (mitoD), and triggering necrosis.

An objective of this research is to demonstrate the feasibility of achieving requisite 4). An early, working hypothesis was that simulated APAP metabolism and toxicity would be essentially the same for virtual culture and mouse experiments. We created a virtual hepatocyte culture and verified that it met requisite 4). We conducted dose-response (D-R) experiments to challenge our working hypothesis. The previously cited virtual experiments (Smith et al., 2016) focused on a dose that maps to $\sim 300 \mathrm{mg} / \mathrm{kg}$ in mice, which causes the characteristic features of APAP-induced liver injury (Saito et al., 2010). The range of our D-R experiments flanked that dose. Because transformation to simulated culture configuration eliminates lobular structures, we expected differences. We were somewhat surprised initially that the two D-R curves crossed because it evidenced that simulated APAP metabolism and toxicity are different for virtual culture and mouse contexts even though individual hepatocyte mechanisms were unchanged. That evidence falsified our working hypothesis. Differences in D-R curves provide a virtual example of an in vivo-to-in vitro disconnect (Fig. 1C).

We use results of experiments to explain the apparent disconnect in detail (Fig. 1D). Individual hepatocytes exposed to the same amounts of APAP respond the same in both contexts. However, the relative contribution of different individual hepatocytes to the system-level phenomenon is different. In liver, hepatocytes are exposed to APAP sequentially, $\mathrm{PP}$ to $\mathrm{PC}$, and both APAP intrinsic clearance and relative production of reactive metabolite are largest (smallest) in PC (PP) hepatocytes; this sequential exposure is absent in culture. An important characteristic of sequential exposure is that relative numbers of hepatocytes are PP $>\mathrm{MZ}>\mathrm{PC}$. Accordingly, hepatocytes from different lobular locations do not respond the same when studied in culture.

We suggest that virtual translational experiments, like those described herein, can be used to begin exploring, untangling, and identifying causes of inaccurate extrapolations. Improved insights can guide selecting additional in vitro measurements to enable more reliable interpretations of in vitro data. This approach provides a new, knowledge- and mechanism-grounded means to begin closing in vivo-to-in vitro disconnects.

\section{Materials and Methods}

Our virtual mouse is engineered to have software components that are concrete and strongly analogous to counterpart mouse components, but only to the extent needed to achieve prespecified targeted 
attributes (Smith et al., 2016). To stress that analogies-although numerous, qualitative, and quantitative-are limited, we refer to a virtual mouse as a Mouse Analog, a virtual culture as a Culture Analog, etc. To limit confusion hereafter and distinguish Mouse (Culture, Hepatocyte, etc.) Analog components, characteristics, and phenomena from mouse (culture, hepatocyte, etc.) counterparts, we capitalized the former. In Results, we describe using the scientific method to falsify three plausible model mechanisms for APAP hepatotoxicity and discover a fourth, which is the one used herein. To support the model mechanism-based explanations of the results that follow, we provide abridged descriptions of Mouse Analog, intraHepatocyte mechanisms, requirements, and technical details.

Mouse Analog. The Mouse Analog, which is illustrated in Fig. 2A, comprises Liver, Body, and a space to contain Dose. Liver, which uses Monte Carlo-determined Lobule variants, is designed and built to be scientifically useful in a variety of usage contexts, including developing model mechanism explanations of drug-induced liver injury. It is engineered to be quantitatively and qualitatively biomimetic during execution and is strongly analogous to actual livers across several anatomic, hepatic zonation, and cell biology characteristics. Having already achieved several qualitative and quantitative target attributes for several different compounds (Yan et al., 2008a,b;
Park et al., 2009, 2010; Smith et al., 2014, 2016), Liver structure and composition (Fig. 2A) are now stable and robust. In this work, a target attribute is a characteristic trait of the liver and APAP metabolism, disposition, and toxicity to which a prespecified similarity criterion is assigned. The latter is a performance requirement. Each wetlaboratory measurement that we seek to mimic (such as the hepatic extraction ratio, clearance, metabolite ratios, and necrosis) becomes a target attribute. A similarity criterion specifies the requisite degree of similarity. An example is that the mean virtual experiment measurement falls within \pm 1 S.D. of the mean wet-laboratory measurement. By increasing the strength and variety of analogies between measurements made during experiments on the Mouse Analog and corresponding measurements made during experiments on mice (left-hand side in Fig. 1D), the credibility of the Liver's multilevel model mechanisms increases.

A Lobule comprises a directed graph with a particular Sinusoidal Segment (SS) object (a software agent) at each graph node. The SS dimensions are Monte Carlo determined within constraints that we refined as we achieved additional target attributes. Graph nodes are organized into three zones. Intrazone edges within zones 1 and 2 (there are none in zone 3 ) mimic interconnections among sinusoids. Numbers of intra- and interzone edges are fixed, but their node-to-node

A A Liver Analog Mouse Analog

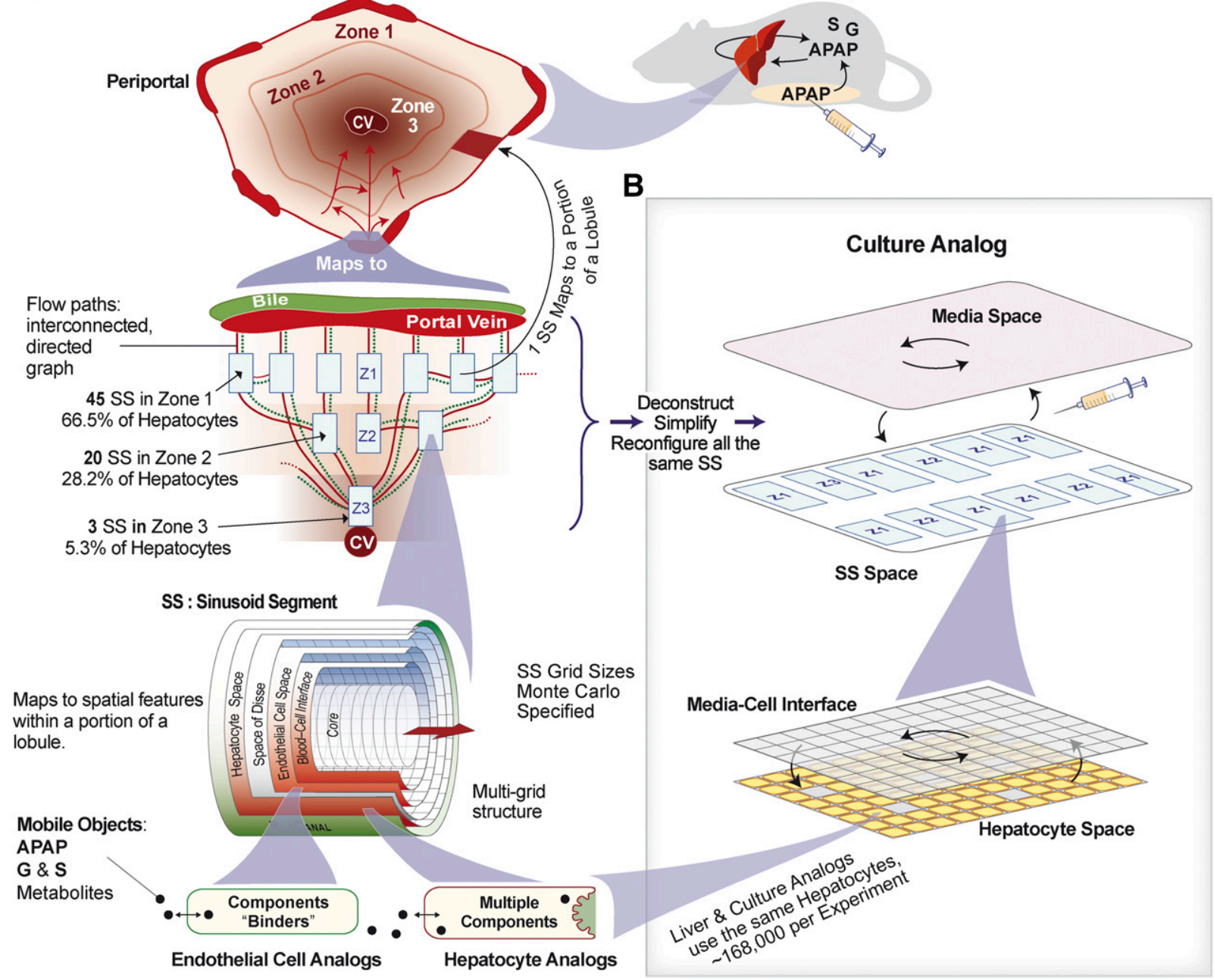

Fig. 2. Mouse and Culture Analogs. (A) A Monte Carlo variant of the Mouse Analog comprises a nested set of all indicated components. Graph structure (flow paths) and dimensions of SS within each variant are Monte Carlo specified. One experiment uses 12 Mouse Analog variants. (B) A Culture Analog comprises Media space and an SS space. All Mouse Lobule SS are first simplified and then placed in SS space. A simplified SS retains its Hepatocyte space and Blood-Cell interface, which are remapped as the Cell-Media interface. Each Culture SS reuses all aHPCs from the Mouse counterpart, and each Culture aHPC reuses the location-specific mechanisms parameterization that it used in the Mouse Analog. Mobile objects enter and exit spaces stochastically. In Culture, they move between Media and the Cell-Media interface, and between the Cell-Media interface and aHPCs. 
assignment is Monte Carlo determined for each execution. All flow paths follow the directed graph. One Lobule maps to a tiny random sample of possible lobular flow paths within a whole liver. Bile (dotted green in Fig. 2A) flows separately from blood (solid red in Fig. 2A) but is not a factor in this work.

Each SS functions as an analog of a random sample of a portion of a sinusoid plus adjacent tissue. It comprises Core, Bile space, and four same-sized grids: the Blood-Cell interface (simply Interface hereafter), Endothelial Cell space, Space of Disse, and Hepatocyte space. Cell objects occupy most of Endothelial Cell (99\%) and Hepatocyte $(90 \%)$ spaces. APAP, its metabolites, and some other Solutes are mobile objects. An APAP object maps to a tiny fraction of an actual APAP dose. A fraction of APAP in Body (along with other mobile objects) is transferred to Portal Vein (PV) each simulation cycle. From there, APAP enters Core and Interface spaces at the upstream end of all zone $1 \mathrm{SS}$. They percolate stochastically through accessible spaces influenced by configuration-controlled local flow. APAP that reaches the distal end of Core and Interface spaces are transferred along a connecting edge to another SS. Mobile objects exit zone $3 \mathrm{SS}$ into the Central Vein (CV), where they get moved to Body.

Solute objects are not limited to representing APAP, its metabolites, and the other molecular entities identified in Fig. 3A. A variety of Solute types, including analogs of drug combinations, can be the focus of Mouse Analog experiments (although doing so was not part of this work). As described previously (Yan et al., 2008a,b; Park et al., 2009, 2010), a particular Solute type can represent any mobile molecule or complex of interest. A Solute's behavior is determined by the properties of its referent, along with the components encountered during a given simulation cycle. An encountered component reads the information carried by the Solute and then uses that information to determine its response utilizing its configuration logic.

Entry and exit of Solutes from each Endothelial Cell and Hepatocyte is mediated by the cell, according to the Solute's properties. Endothelial Cells contain Binders that bind and release APAP (maps to nonspecific binding). There are $\sim 14,000$ Hepatocyte Analogs (aHPCs) per Lobule. They use previously validated event management modules (Petersen et al., 2014) to control material entry and removal along with binding and the object transformations described in Fig. 3. The order of events is (pseudo) randomized each simulation cycle. Mouse Analogs, and thus all Hepatocytes, are currently gender neutral.

Culture Analog. Petersen et al. (2016) used a similar Mouse Analog along with 2D models of hepatocyte cultures to explore and challenge mechanism-based hypotheses about immune-mediated cytochrome P450 downregulation in vitro. The aHPCs used in the two systems contained the same internal components. However, because Petersen et al. (2016) parameterized aHPC separately, they simulated rather than explained the in vivo-to-in vitro disconnect (Fig. 1C) within the validation data.

Culture Analog is illustrated in Fig. 2B. Culture reuses all Mouse Analog aHPCs. Culture uses Media space and SS space. Media space mimics a well-mixed system. Culture does not use the Lobule's directed graph. All SS from a Lobule (along with all aHPC within) are simplified, reinstantiated, and then placed into Culture's SS space. Culture does not use Core and Bile spaces, nor does it use Endothelial Cell and Disse grids. It reuses aHPC and Interface grids. We remap the latter as the Media-Cell interface. Each simulation cycle, a small fraction of APAP (and other specified Solutes) in Media, is transferred randomly to each Media-Cell interface grid. From there Solutes move within Hepatocyte space and enter and exit aHPCs exactly as they do within Mouse Analog Lobules. Except when specified otherwise, each Culture aHPC reuses the same locationspecific mechanism parameterization (Fig. 3B) that it used when it functioned within Mouse Analog. For the same experimental objective, differences in wet-laboratory culture architectures can contribute to differences in experimental outcomes. The same is true for Culture Analogs.
Intra-Hepatocyte Mechanisms. Objects within aHPCs and their capabilities are identical to those used previously (Smith et al., 2016). The event descriptions that follow are per simulation cycle. Hepatocytes contain four types of physiomimetic modules (Petersen et al., 2014): InductionHandler (not used in this work), EliminationHandler, MetabolismHandler, and BindingHandler.

There is a direct mapping between the probability of an APAP metabolism event (for each APAP in each aHPC for each second) and amounts of metabolic enzymes. Both the probability of an APAP metabolic event and the probability that the Metabolite is $N$-acetyl- $p$ benzoquinone imine (NAPQI) increase 3 -fold from the PV entrance to the CV exit. All other metabolites are lumped together and divided equally between Glucuronide and Sulfate (maps to the glucuronide and sulfate metabolites, labeled as G\&S in Fig. 3B). A Mechanism uses particular values from each of the Fig. 3B gradients (from the PV entrance to the CV exit). Each gradient is implemented explicitly as a function of distance from the PP entrance to the aHPC's position. The APAP Liver extraction ratio measured at steady-state (with constant rate APAP infusion) averages 0.5.

With probability to react $=0.5$, each NAPQI undergoes a reaction. At early times, each NAPQI reaction decrements the aHPC's GSH Depletion threshold value by 1.0 , which maps to depleting a fraction of a hepatocyte's available GSH. Each aHPC has a location-determined GSH Depletion threshold value. A small or zero threshold value means that aHPC is most sensitive to NAPQI-caused hepatotoxicity. After the GSH Depletion threshold is breached, NAPQI reacts to form one of two types of Damage products. Adhering to a strong parsimony guideline, we specified these Damage products as follows: mitochondrial damage products (maps to mitoD), and nonmitochondrial damage products (maps to nonMD, or all other types of damage).

The downstream resolution of events triggered by Damage products is inadequate to simulate toxicity phase events. Mindful of our strong parsimony guideline, we specified that one NAPQI $\rightarrow(1+n)$ mitoD, where $n$ is a pseudo-random draw from uniform ( 1 to 6 ) hours. MitoD amplification also maps to the accumulation of reactive oxygen/ nitrogen species. Cell death (Necrosis) is triggered when the amount of mitoD is greater than the Necrosis Trigger threshold value. Once an aHPC is designated dead (Necrotic), it stops metabolizing APAP.

Cell death always follows a Necrosis Trigger event. Because necrosis is a process, there is a delay between the triggering event and when necrosis becomes detectable in stained tissue sections. Death Delay maps to that process. Thus, the time of a detectable aHPC death $=$ time of trigger event + a pseudo-random draw from uniform (1.2 to 12 ) hours.

Hepatocytes use multiple mechanisms to mitigate or reverse different types of damage. Consistent with our strong parsimony guideline, we implemented a single mitigation Mechanism, repurposing a Metabolism Module, and named it Repair. It maps to a conflation of all actual mitigation/recovery mechanisms. With a locationspecified probability, a Repair object replaces a Damage product. We focused on mitoD because only it can trigger Necrosis. By specifying that the probability of a mitoD Repair event decreases sigmoidally from the PV entrance to the CV exit, we enabled Necrosis Trigger events to occur first close to the CV, which was the key targeted attribute in Smith et al. (2016).

Requirements. We use the virtual experiment approach described by Kirschner et al. (2014) along with the enhanced strategies detailed by Petersen et al. (2016) and Petersen and Hunt (2016). The model mechanisms and methods require meeting the following four requirements.

1. Mouse and Culture Analogs during execution must exhibit all five primary characteristics of a biologically explanatory mechanism (Darden, 2008): 1) the mechanism is biomimetic and responsible for the phenomena; 2) the mechanism has components: modules, entities, and activities; 3) components are arranged spatially and can exhibit structure, localization, orientation, connectivity, and compartmentalization; 
A
A

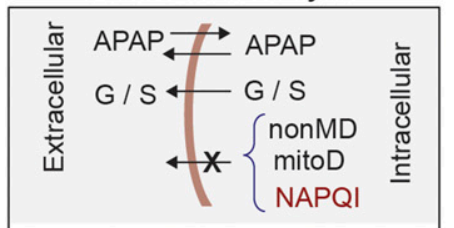

APAP Free $\rightleftarrows$ APAP Bound

APAP Bound $\longrightarrow$ Metabolism Event

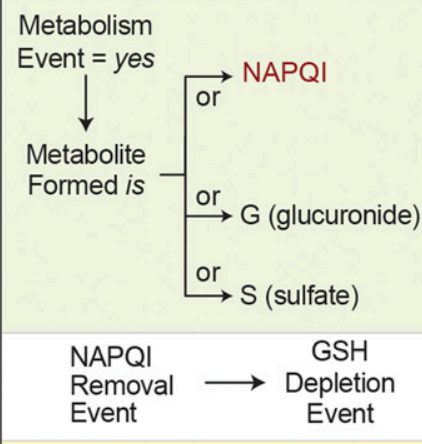

GSH Depletion Threshold

\section{When GSH Depletion Threshold Value $=0$

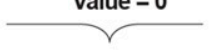

Formation of damage products
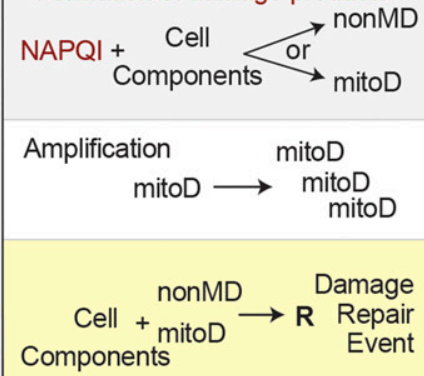

Components

$$
\text { mitoD }>\underset{\text { Threshold }}{\text { Necrosis }} \rightarrow \underset{\text { Triggered }}{\text { Necrosis }}
$$

Necrosis becomes detectable following a randomly selected Delay
B

\section{Location-Dependent \\ Parameter Values}
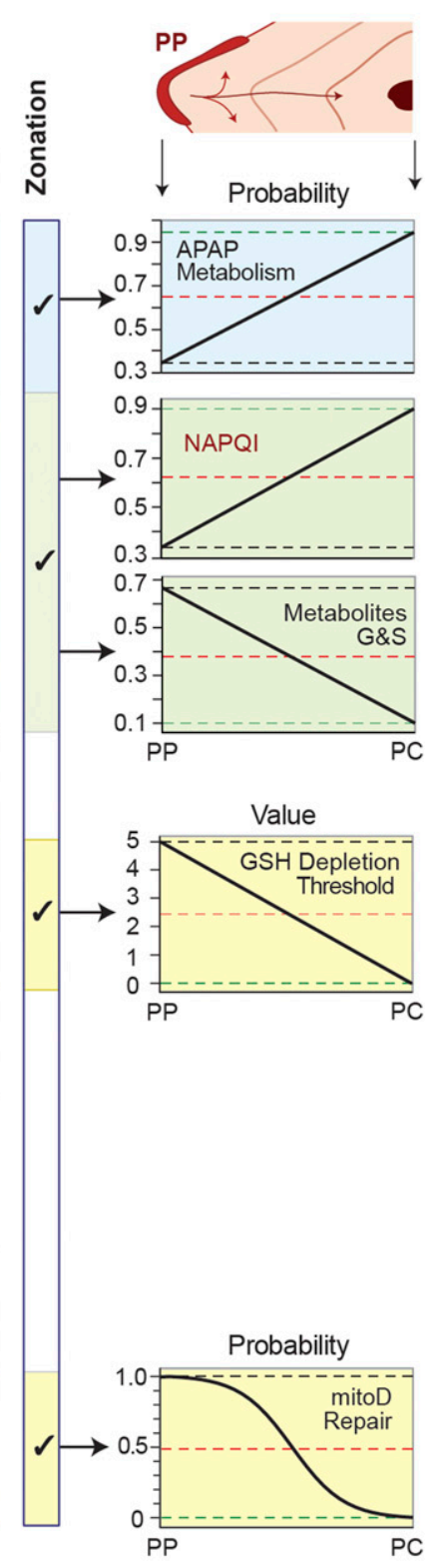

Threshold Value $=5$ mitoD
Fig. 3. Intra-Hepatocyte events in Mouse and Culture Analogs. (A) Listed under Hepatocyte are the events and activities described in the text. Each event can occur within each aHPC in each simulation cycle, as detailed in Smith et al. (2016). Each event executes independently in a pseudorandom order. All events are stochastic. (Right-hand side) The check marks identify events that are subject to zonation. Prior to GSH Depletion, there is an approximately $10 \%$ chance that the NAPQI removal event will result in creation of a Damage product. Thereafter, each NAPQI removal event results in creation of a Damage product. When Necrosis is triggered, it becomes measurable (maps to cells that stain positive for necrosis) in the future after some parameter-specified number of simulation cycles. (B) The five graphs show how these events depend on the Lobular location. (Left-hand side value) The PP value assigned to aHPCs located at SS grid
4) activities have temporal aspects including rate, order, duration, and frequency; and 5) the mechanism has a context, which can include being in a series and/or a hierarchy.

2. Components and spaces (Fig. 2) are concrete, biomimetic (Pogson et al., 2006; Hunt et al., 2011), and sufficiently modular to facilitate analogical reasoning (see http://plato. stanford.edu/archives/fall2013/entries/reasoning-analogy/ and http://plato.stanford.edu/archives/fall2012/entries/models-science/).

3. Phenomena measured at a higher level or layer of organization arise mostly from local component interactions at a lower level of organization.

4. Each mobile object type maps to a particular chemical entity. Quasi-autonomous components (i.e., software agents such as SS and aHPCs) recognize different mobile objects and adjust their response appropriately. For example, an aHPC recognizes that an adjacent object has the property membrane crossing $=$ yes, and allows it to enter stochastically.

To achieve requirement 2, Mouse Analogs are written in Java, utilizing the MASON Multiagent Simulation Toolkit (Luke et al., 2005).

Analog Technical Details. Analogs are treated as a form of data, using both the implicit schema of Java, JavaScript, and $R$ and the explicit schema of the configurations. Mouse Analogs and configuration files are managed using the subversion version control tool in two repositories, one private (Assembla, San Antonio, TX) and another public. The data presented herein along with Mouse and Culture Analog codes are available from project websites (https://simtk.org/ projects/aili and https://simtk.org/home/isl/).

The entire toolchain, including the operating system, configurations, and input/output handling is open source. All project-generated released data are available to be licensed as open data. We execute virtual experiments using local hardware and in a cloud environment. Experiments described herein were run using local hardware and virtual machines (Ropella and Hunt, 2010) on Google Compute Engine, running 64-bit Debian 7. Analog quality assurance and control details are discussed in Smith et al. (2016) along with practices followed for validation, verification, sensitivity analyses, and uncertainty quantification.

Homogeneous Culture Analogs. The canonical experiment is the isolation of hepatocytes from a liver lobule followed by transfer to culture plate wells. An assumption in this process is that the proportion and types of hepatocytes obtained from the different lobular zones are the same when grown in culture (see Fig. 2). However, this assumption may not be true; therefore, we did virtual experiments in which all Hepatocytes in the Culture systems were configured to approximate a situation wherein they had been obtained from the same zone, either near the CV, MZ, between PV and CV, or near the PV. Specifically, the reaction probabilities and GSH Depletion thresholds were held to constant values representative of each region. For these experiments, Culture Analogs were dosed with same APAP amounts used in previous experiments. However, intra-Hepatocyte Mechanisms were configured with no zonation. These configuration changes are illustrated graphically in Fig. 3B as horizontal dashed lines, which represent no zonation.

\section{Results}

The Model Hepatotoxicity Mechanism under Study. In this section, we summarize the key evidence from Smith

spaces adjacent to the PV entrance. (Right-hand side value) The PC value assigned to aHPCs located at SS grid spaces adjacent to the CV exit. The black, red, and green dashed lines specify Lobular location-independent values used by aHPCs in the three homogeneous Hepatocyte Cultures (see Fig. 7). 
et al. (2016) that supports our working hypothesis that actual temporal details leading to centrilobular hepatotoxicity in mice following APAP dosing are strongly analogous-quantitatively similar-to details occurring during execution of Mouse Analogs having the Hepatocyte parameter values illustrated in Fig. 3. The target phenomenon in mice is that necrosis is first triggered close to the lobule's $\mathrm{CV}$ and progresses outward thereafter. Examples of histologic evidence of the characteristic dose-dependent PC necrosis are provided in Mitchell et al. (1973), Roberts et al. (1991), Anundi et al. (1993), Hinson et al. (2010), and Ni et al. (2013). Ample evidence (e.g., Anundi et al., 1993) supports that zonation of NAPQI formation within lobules, as depicted in Fig. 3, is a necessary and sufficient requisite to account for that phenomenon. In Smith et al. (2016), we refer to that explanatory mechanism as the NAPQI zonation (NZ) Mechanism. Challenging that mechanism in mice is problematic because 1) variables determining causes and effects cannot be controlled adequately, and 2) it would require sequential intracellular measurements at different lobular locations within the same mouse. We circumvented those impediments using virtual Mouse Analog experiments.

The NZ Mechanism used only the top three of the five parameter zonations shown on the right-hand side in Fig. 3. When using a constant GSH depletion threshold of 3.0 and a constant probability (for each mitoD within each aHPC for each second) of mitoD repair of 0.6, Mouse Analogs using the NZ Mechanism achieved quantitative validation targets for APAP clearance and metabolism but clearly failed to achieve the target phenomenon because early Necrosis Trigger events occurred first in the PP and MZ regions. Consistent with our strong parsimony guideline, we posited that to achieve the target phenomenon at least one additional model mechanism feature must exhibit zonation. We explored two options: zonation of 1) GSH Depletion threshold values and 2 ) mitigation of mitoD.

We first explored having the GSH Depletion threshold diminish in the $\mathrm{CV}$ direction, as illustrated in Fig. 3. We refer to the resulting configuration as the GSH NZ (GNZ) Mechanism. Such zonation of GSH Depletion is supported by evidence that GSH levels are higher in PP than in PC hepatocytes (Sastre et al., 1992). The early PP Necrosis Trigger events observed with the NZ Mechanism were eliminated; however, average early Trigger events occurred Midzonally (not PC), thus the GNZ Mechanism was also insufficient.

Mitigation of mitochondrial damage is known to play a role in recovery from drug-induced liver injury, but to date, to our knowledge, no one has sought wet-laboratory evidence that mitigation of mitochondrial damage does or does not exhibit zonation. However, GSH replenishment capacity, which may be a feature of mitigation of mitochondrial damage, is lower in PC than in PP hepatocytes (Kera et al., 1988). We tested the hypothesis that, in Mouse Analogs, a linear reduction in the probability of mitoD mitigation events in the CV direction (similar to the zonation pattern for the GSH Depletion threshold) combined with the NZ Mechanism (but absent zonation of the GSH Depletion threshold) would achieve the target phenomenon. The temporal pattern for location of average Necrosis Trigger events was closer to the CV than that for the NZ Mechanism, but the improvement was insufficient. Changing from a linear zonation pattern to the sigmoidal pattern shown in Fig. 3 resulted in further improvement, but it also failed to achieve the target phenomenon because the earliest Trigger events were still occurring Midzonally. The latter configuration is the MNZ Mechanism.

We conjectured that merging the MNZ and GNZ Mechanisms would enable achieving the target phenomenon, and it did. We argue that, at corresponding degrees of granularity, the merged MNZ and GNZ Mechanism and actual intrahepatocyte mechanisms in mice are strongly analogous within and across multiple lobular levels. It is noteworthy that we discovered that variants of the MNZ and GNZ Mechanism provide plausible quantitative explanations for the considerable variation in 24-hour necrosis scores among 37 genetically diverse mouse strains following a single toxic APAP dose (Smith et al., 2016).

Dose-Response Curves. We use the occurrence of Necrosis Trigger events as our measurement of response. In both Mouse and Culture Analogs, cumulative Trigger events reach a plateau within 180 minutes. We conducted D-R experiments (12 Monte Carlo variants). During each experiment, we administered identical doses of APAP ranging from 6000 to 500,000 (largest APAP dose) objects per experiment. Figure 4 displays the results. Dose-dependent differences were also observed (data not shown) in measurements of other attributes. Differences in occurrence of Necrosis Trigger events between Mouse and Culture experiments are a complex consequence of differences in aHPC exposure to APAP and aHPC heterogeneity (discussed subsequently). In Culture, the

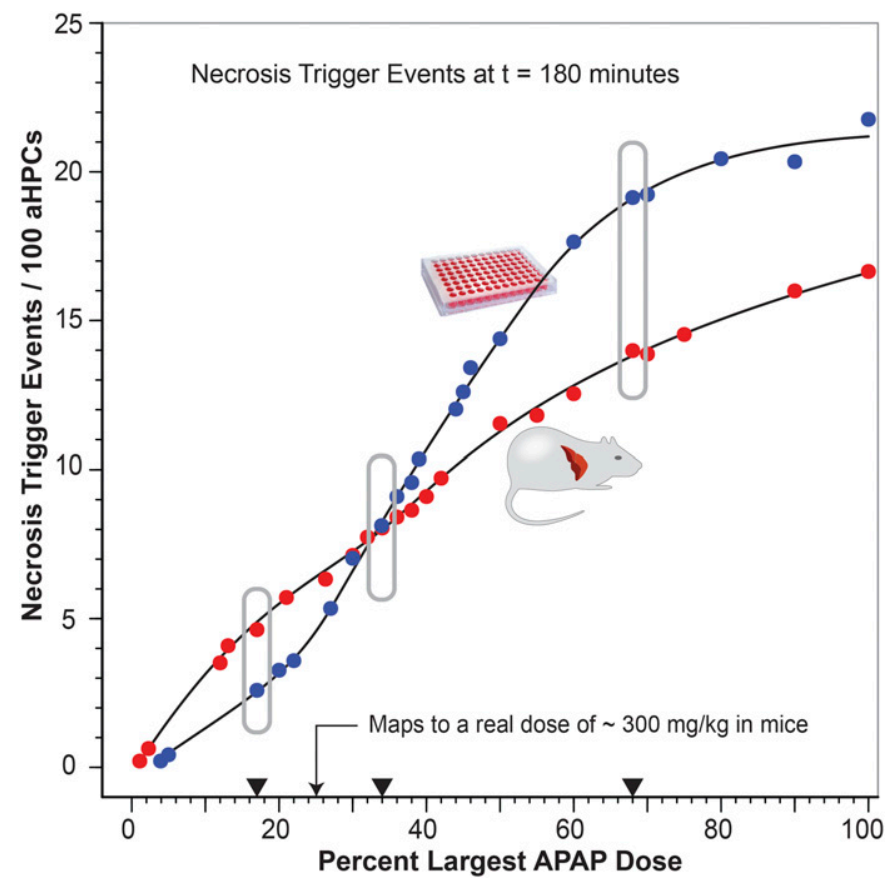

Fig. 4. Dose-response curves for Mouse and Culture Analogs. Dose range: 6000-500,000 APAP objects per variant. Experiments were conducted separately using different local computers and cloud virtual machines and with different sets of random number seeds. The indicated simulated dose is the same as that used in Smith et al. (2016), which maps to a real dose of $\sim 300 \mathrm{mg} / \mathrm{kg}$ in mice and is the most frequently used dose for studying APAP-induced liver injury in mice (Saito et al., 2010). By 180 minutes, in all experiments, cumulative Necrosis Trigger events (response) are close to-or have reached - their plateau values. Larger doses (e.g., $600 \mathrm{mg} / \mathrm{kg}$ in many strains) can be simulated, even though they can be lethal. Figures 5 and 6 present measurements of intra-Hepatocyte phenomena at the three indicated doses. 
organizational structure of Lobules is absent. Culture aHPCs are exposed to APAP that, on average, is distributed uniformly within the media-cell interface grids. However, within a Mouse Lobule aHPCs are exposed to APAP sequentially. During a given interval, upstream and downstream aHPCs are "seeing" different amounts of extra-Hepatocyte APAP. In Culture, that sequential exposure to xenobiotics is absent. Differences in exposure dynamics between Mouse and Culture Analogs alter intra-Hepatocyte phenomena. The different D-R curves in Fig. 4 are a consequence.

Hepatocyte-Level APAP Disposition and Toxicity Measurements. At the end of each simulation cycle (1 second), we measured the number of Necrosis Trigger events. Virtual measurements are made analogous to wet-laboratory counterparts to facilitate comparisons when wet-laboratory data are available. Crossing D-R curves indicate that mechanism entities and activities are contributing differently to cumulative Necrosis Trigger events from smallest to largest dose. To help identify and explain those differences, measurements of selected phenomena, averaged over all Liver aHPCs, are plotted in Fig. 5. Simulated intraperitoneal dosing causes peak APAP amounts in Mouse to occur later than in Culture. For each row of plots in Fig. 5, compare the temporal profile in Mouse to that in Culture for each dose. Cumulative Necrosis Trigger events in Mouse are greater than in Culture at the smallest dose, but that relationship is reversed at the largest dose. For APAP in aHPCs, the Mouseto-Culture profile relationships are about the same for all
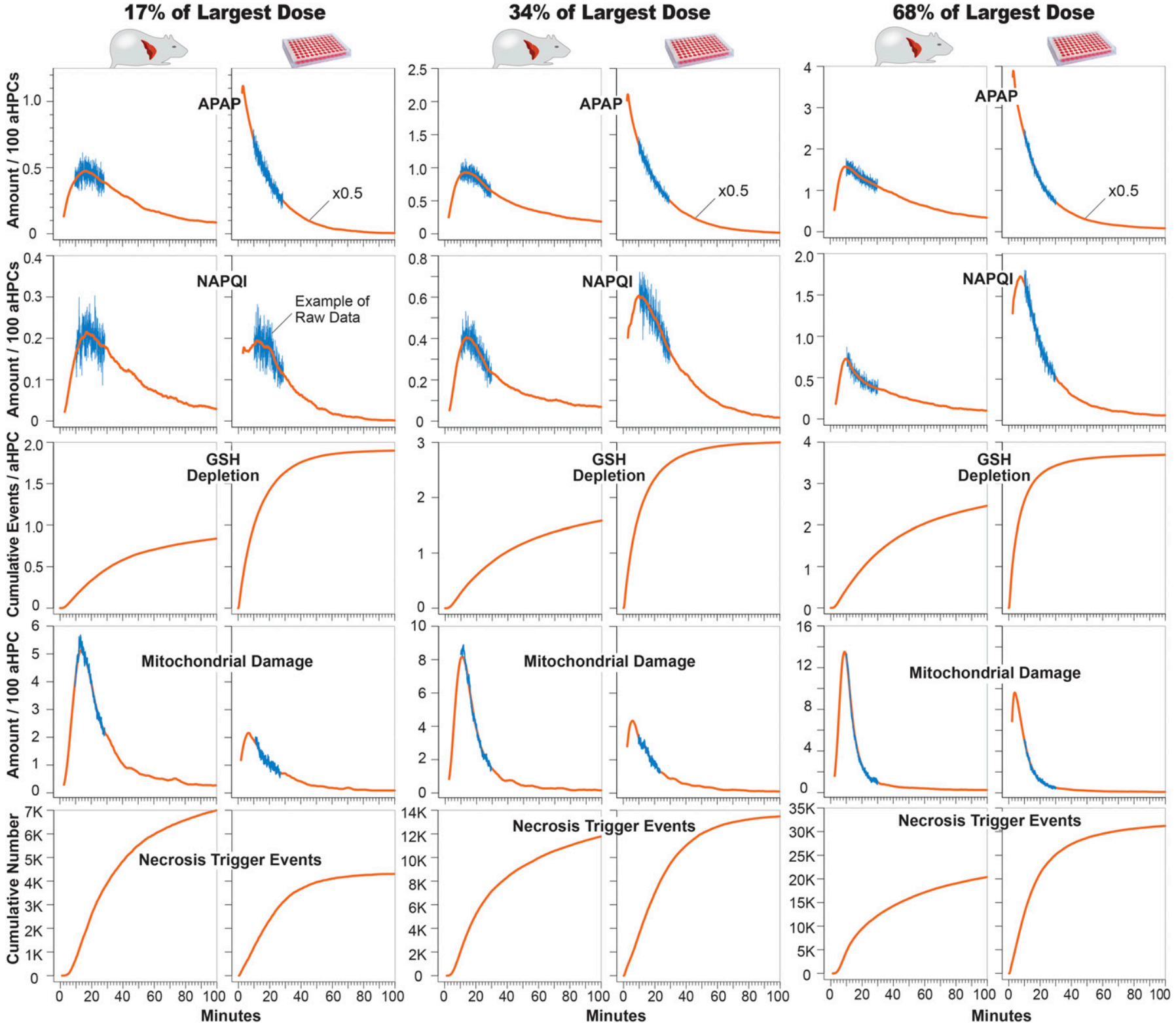

Fig. 5. Selected phenomena in Mouse and Culture Analogs. Each row displays temporal profiles for relevant phenomenon measured each second during the Mouse and Culture experiments at the three doses indicated in Fig. 4. The software configuration values for each aHPC are the same for Mouse and Culture. The range of $y$-axis values increases with increasing dose. Values for amount/100 aHPCs are 101-point centered averages. The actual per second values between 10 and 30 minutes are plotted in blue to illustrate within-experiment variance. Relative variance for smaller-dose experiments is larger because there are fewer objects and fewer events. The corresponding variance is not evident in cumulative values; however, a repeat experiment would not generate the exact same cumulative value profile. Largest dose: 500,000 APAP per execution; 12 executions/experiment. 
three doses, which is not the case for NAPQI, GSH Depletion events, and mitoD.

At comparable times, the differences in NAPQI amounts in Culture aHPCs, relative to those in Mouse, increase with increasing APAP dose. We see a similar trend for mitoD. However, that trend is reversed for GSH Depletion events. At the smallest dose, relative to results in Culture, fewer Mouse GSH Depletion events caused a larger number of Necrosis Trigger events. That observation seems counterintuitive. At the largest dose, we do not see that difference: for both mouse and culture, the relationship between cumulative GSH Depletion events and cumulative Necrosis Trigger events is similar. Explanations of those results, including the counterintuitive observation, require the more detailed, Lobular location-dependent information provided in Fig. 6.

Amounts of APAP, NAPQI, and mitoD per aHPC, along with GSH Depletion events were measured separately in SS in zones 1, 2, and 3. We measured Trigger events in PC, MZ, and PP regions (defined in the Fig. 6 legend) and plotted them in Fig. 6. For Mouse, values for APAP and GSH Depletion in zone 3 during the first 10 minutes are largest for all three doses, whereas in Culture the zone 1 values are largest. That striking difference highlights Mechanism differences between aHPCs in Mouse and Culture and contributes to an explanation of the virtual Mouse-Culture disconnect. That difference is a consequence of two factors: 1) the sequential versus simultaneous
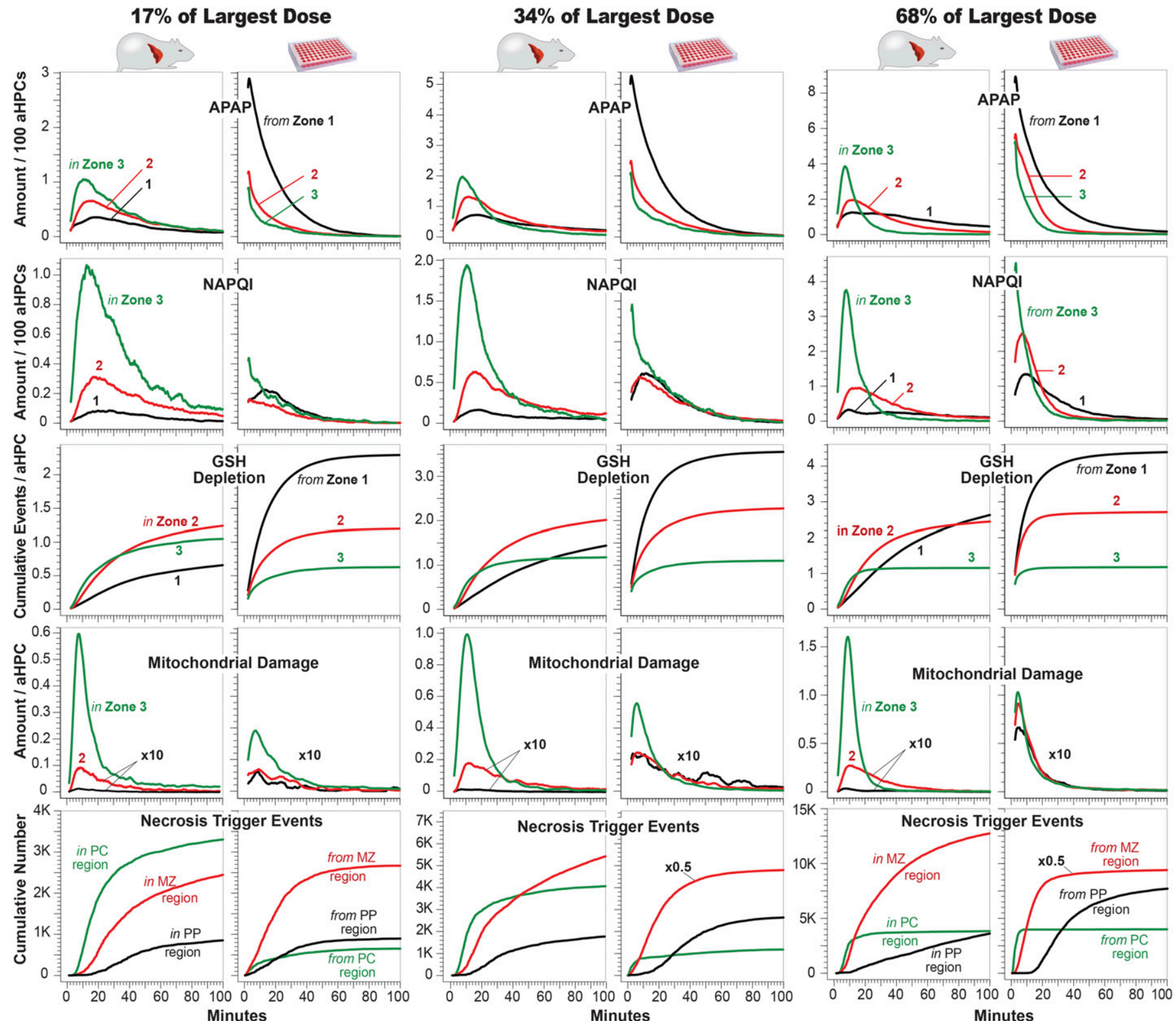

Fig. 6. Hepatocyte-level attributes in Mouse and Culture Analogs by zone. The data are from the same experiments as in Fig. 5. Except for Necrosis Trigger events (see the text), measurements are plotted for aHPCs in each SS zone (e.g., in zone 3, 2, or 1) for Mouse. For Culture, zone refers to the location of aHPCs within the Lobule before they were isolated (e.g., from zone 1, 2, or 3) and reconfigured (Fig. 2B) into the Culture Analog. By measuring average values per SS, we can compare how similarly configured aHPCs in the Mouse and Culture contexts behave during exposure to APAP. Cumulative number of Trigger events: PC (location is $<10$ grid spaces from the CV exit); MZ ( $10<$ location is $<20$ grid spaces from the CV exit); and PP $(20<$ location is $<30$ grid spaces from the CV exit). The range of $y$-axis values increases with increasing dose. Values for amount/100 aHPCs and average Trigger event locations are 101-point centered moving averages. 
aHPC exposure described previously, and 2) the majority of Lobular aHPCs are in zone 1. For each Lobule variant, there are 45 SS in zone 1, 20 SS in zone 2 , and three SS in zone 3. That structure maps directly to the polyhedral nature of lobules. Compounds in blood entering PV tracts get exposed to many more hepatocytes than blood exiting the CV. In Liver analogs, the zone 1 /zone 3 aHPC ratio averages about 12.5 , with $66.5 \%$ of aHPCs in zone 1 and $5.3 \%$ in zone 3 . Consequently, APAP/aHPC in zone 3 is greater than in zones 2 and 1. The ratio would be larger except for a mitigating factor: as specified in Fig. 3B, the probability of an APAP Metabolism event is zone $3>$ zone $2>$ zone 1 ; zone 3 aHPCs are drained of APAP faster than zone 1 aHPCs. In Culture, one might expect APAP/aHPC to be about the same because unbound APAP is directly proportional to local APAP amounts within the Media-Cell interface grid. However, APAP/aHPC values for zones 2 and 3 are smaller primarily because the probability of an APAP Metabolism event is larger in zones 2 and 3 than in zone 1.

Because NAPQI formation increases PP-to-PC (Fig. 3B), the amounts of NAPQI/aHPC in Mouse are largest in zone 3; only at early times is the same true for Culture. At comparable times after 20 minutes, compare Mouse-to-Culture NAPQI/ aHPC at each dose. For the smallest dose, the values for zones 2 and 3 are larger for Mouse. For the largest dose, the values for zones 1 and 2 are greater for Culture. That reversal is caused primarily by a combination of two factors: the sequential versus simultaneous aHPC exposure and by 10 minutes, all zone 3 aHPC in both Mouse and Culture have experienced Necrosis Trigger events.

By 30 minutes after the small APAP dose, Mouse GSH Depletion events in zone 2 begin exceeding those in zone 3 . That transition is a consequence of two factors: 1) as plotted in Fig. 3B, average GSH Depletion threshold values are zone $2>$ zone 3, thus more GSH Depletion can occur in zone 2; and 2) Depletion events accumulate more slowly in zone 2 because less APAP is Metabolized to NAPQI in zone 2 relative to zone 3. In Culture, cumulative Depletion events are largest for zone 1 because Depletion threshold values for zone 1 aHPCs are largest; there is more GSH to deplete.

For Mouse and Culture, the relative per zone profile patterns for the amount of mitoD/aHPC are similar to those for NAPQI except that the amount of mitoD/aHPC in zones 2 and 3 are lower than in zone 3 by more than $10 \times$. This large difference is because of greater rates of Damage Repair in zones 1 and 2 , which limit the accumulation of mitoD.

To see why the D-R curves cross, first consider the high dose cumulative Trigger event profiles. Within 30 minutes, Necrosis is triggered in all PC aHPCs in Mouse and Culture. Because of the more rapid PP Depletion of Culture GSH relative to Mouse GSH, Trigger events accumulate faster in Culture in PP aHPCs. By 60 minutes, Necrosis has also been triggered in all MZ aHPCs in Culture, whereas in Mouse the MZ aHPCs values are still increasing. The situation is entirely different for cumulative Trigger event profiles following the small dose. In Mouse, PC aHPCs, which are most sensitive to NAPQI toxicity, experience greater APAP exposures than do upstream aHPCs. That is not the case in Culture. Relative to Mouse, exposure per aHPC is the same in Culture.

For Mouse, at all three doses, early Trigger events occur in the $\mathrm{PC}$ region close to the $\mathrm{CV}$ exit. At $t=100$ for the smallest dose, MZ Trigger events in both Mouse and Culture are similar, yet in Mouse, Trigger events are even larger in PC aHPCs, whereas in Culture, they are smallest. Furthermore, Necrosis in Mouse has been triggered in most PC aHPCs, whereas in Culture Trigger events have occurred in only a fraction of PC aHPCs. These differences contribute to an explanation of the virtual Mouse-Culture disconnect (Fig. 1C).

Homogeneous Culture Analogs. It is evident from Fig. 6 that PP MZ, and PC aHPCs behave differently in Culture than they do in Mouse. Given that, the following is a logical query, even though corresponding wet-laboratory experiments are infeasible. How would the measured features be different if identical experiments were conducted using Cultures comprising only aHPCs that are isolated from a particular Lobular location, such as adjacent to CV or PV in Mouse Analogs, or somewhere in between? Responding to that query, we conducted experiments, identical to those in Figs. 5 and 6, in which all aHPCs were parameterized to approximate a situation wherein they had been obtained from one of three locations: 1) PP, adjacent to the PV entrance; 2) PC, adjacent to the CV exit; or 3) MZ, approximately halfway between 1) and 2). Parameter values for the aHPCs are specified by the three dashed lines in Fig. 3B, where black specifies PP, red specifies MZ, and green specifies PC aHPCs.

Four sets of attribute measurements are presented in Fig. 7. The influence of zonation on attribute profiles that is evident in Fig. 6 is amplified in these homogeneous Cultures. The most striking observation is that for homogeneous PC aHPCs. Necrosis is triggered within 60 minutes in essentially all aHPCs for the middle and largest doses. There are dramatically fewer Trigger events in corresponding experiments utilizing homogeneous MZ and PP aHPCs. Also, Trigger events in Cultures of PP aHPCs accumulate slowly because the large probability of a Repair event hinders accumulation of GSH Depletion events.

\section{Discussion}

Biomimetic software mechanisms become scientifically interesting and useful analogies when temporal measurements of generated phenomena are similar to wet-laboratory counterparts, within some prespecified tolerance. The Liver analog, components within aHPCs (Fig. 3), and the events used to simulate features of APAP hepatotoxicity are analogous to an actual liver, hepatocytes, and pharmacodynamics, but only to the extent needed to achieve prespecified targeted attributes (Smith et al., 2016). The complemental methods presented herein use virtual experiments to measure changes that occur when all aHPCs from a Mouse Analog's Liver are precisely translated into a $2 \mathrm{D}$ configuration, which is strongly analogous to hepatocytes in $2 \mathrm{D}$ cultures. Within a Mouse Lobule, APAP exposure to aHPCs is somewhat sequential. Consequently, upstream and downstream aHPC exposures are different amounts. The different exposures depend on the size of dose. For a small dose, more PV cells are exposed because APAP starts to percolate through the sinusoids near the PV, decreasing the amount available near the CV; however, for a large dose exposure across all zones approach the same amount because the amount of APAP available that percolates through the sinusoids is enough to eventually saturate the cells. Therefore, a relevant relational measure is the amount of APAP per cell, which is low near the PV and high near the CV because there are more PV cells than CV cells. In Culture, 

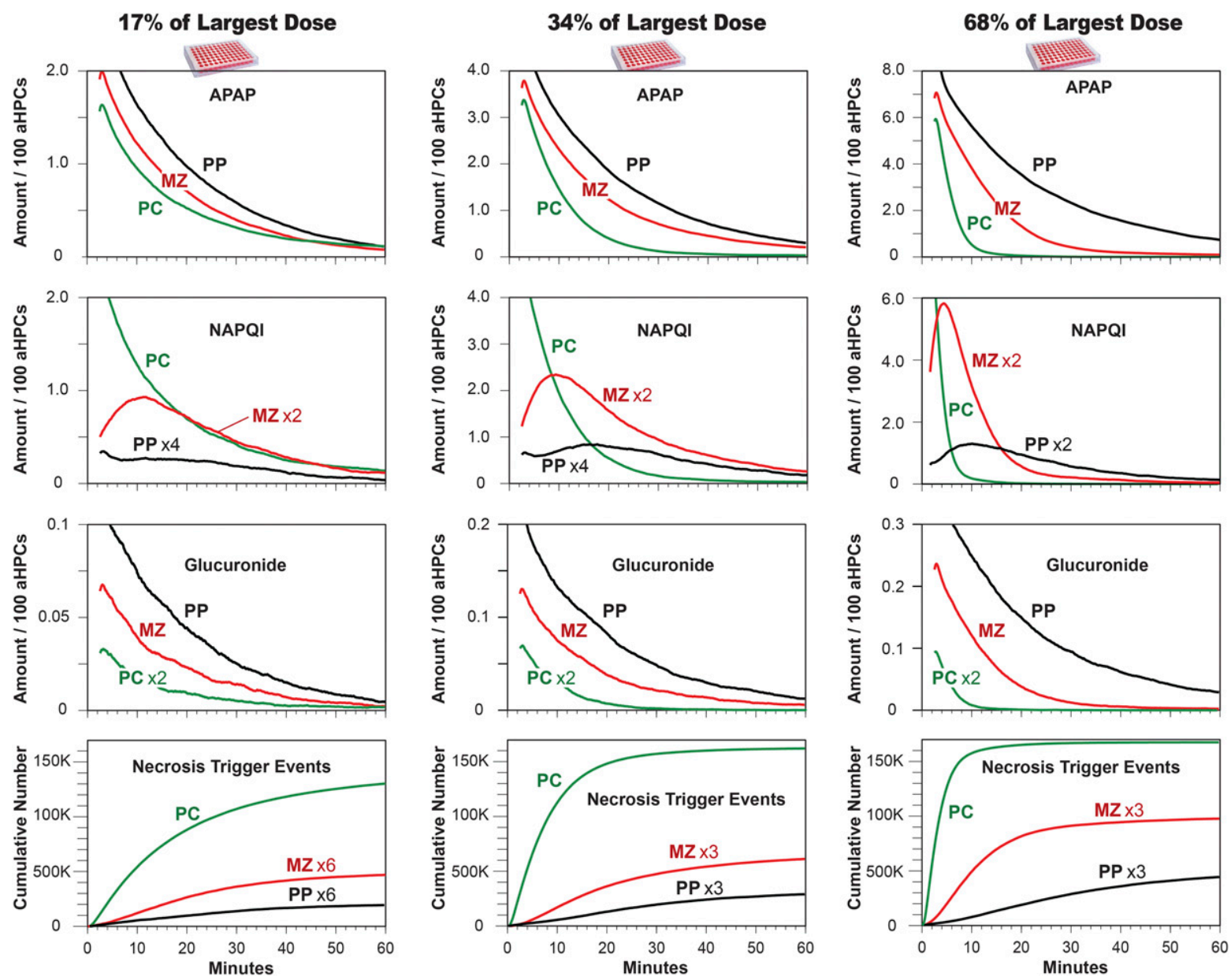

Fig. 7. Selected phenomena observed in three homogeneous Hepatocyte Cultures. The experiment protocol is the same as for Figs. 5 and 6 . Culture structure, SS, and aHPC numbers are simple Monte Carlo variants of those for Figs. 5 and 6 . However, the location-dependent parameter values illustrated in Fig. 3B (solid black) have been replaced by constant, Lobular location-independent values. PP Culture: parameter values are specified by the dashed black lines in Fig. 3B. MZ Culture: the dashed red lines specify parameter values. PC Culture: the dashed green lines specify parameter values.

because the organizational structure of Lobules is absent, that sequential exposure to APAP is absent. APAP exposure to aHPCs is essentially uniform, and the amount of APAP per cell remains constant. These differences in exposure dynamics between Mouse and Culture Analogs alter intra-Hepatocyte phenomena. The different D-R curves in Fig. 4 are a consequence. We learned more about that Mouse-Culture disconnect by comparing the similarities and differences in how a variety of attributes (Figs. 4-6) respond to identical simulated doses of APAP.

The approach was developed with the expectation that it can be generalized. For any xenobiotic or biologic of interest, consistent with current knowledge, Mouse and Culture Analogs (everything in Fig. 2) can be reused without change to begin studying and improving mechanism-based explanations for hepatic disposition and metabolism phenomena along with the earliest stages of hepatotoxicity for those compounds where hepatotoxicity is or may be an issue. However, for improving mechanism-based explanations of hepatotoxicity, the events in Fig. 3 would need to be reworked and reconfigured to be consistent with what is known about early toxicity details for each compound of interest.
We envision information obtained during Mouse-Culture comparisons being used to explore plausible explanations for a known or suspected wet-laboratory disconnect. The approach may also prove useful for incrementally developing challengeable theories about mechanism features lost and gained during transition from whole organism to final in vitro configuration (Fig. 1B).

To fully understand the behaviors of hepatocytes in a wetlaboratory experimental context, we must take into account various relevant contexts in which they function to generate phenomena of interest, and how function and phenomena change according to context. To advance that understanding, we must consider the methods and processes used to engineer an in vitro (and other) experimental system from whole animal sources. The difficulty of the task is increased by changes in hepatocyte states during isolation that are challenging to document and control. The information needed to begin closing or bridging an in vivo-to-in vitro disconnect cannot be obtained using wet-laboratory methods alone because complete hepatocyte behavior cannot be measured either in vitro or in vivo. The methods used herein are intended to contribute to the larger effort. We suggest that one can begin bridging that disconnect by hypothesizing, 
instantiating, and then exploring corresponding plausible model mechanisms for Culture and Mouse contexts as illustrated in Fig. 1D.

We embedded Model Mechanism knowledge along with structural information within Liver and Culture Analogs. Comparisons of results from virtual experiments on both Analogs are expected to help identify and explain observed differences between in vitro and in vivo systems. In many cases, bridging wet-laboratory disconnects will be considerably more challenging than bridging a virtual disconnect, so it is instructive to first consider how the latter might be accomplished. Given only the Culture data in Figs. 4 and 5, but no details about the Culture Mechanism, we face a virtual in vivo-to-in vitro disconnect. For example, it is infeasible to predict (within some reasonable tolerance) cumulative Necrosis Trigger events within Mouse Analogs, even though the Culture data in Fig. 5 are quite detailed. Even knowing that experiments in Figs. 4 and 5 met the fourth requisite in the Introduction (i.e., same intra-Hepatocyte Mechanisms), we need some measure of aHPC homogeneity, or lack thereof, in Culture to begin constructing a Mechanism-based hypothesis to bridge across the disconnect. The following is an example of a virtual measurement of aHPC heterogeneity: create nonmembrane-crossing mobile reporter objects. Include them with the APAP dose. When adjacent to an aHPC, a reporter object asks the aHPC, is the value of your GSH Depletion threshold $\geq 2$ ? If no, the reporter takes on a value of 0 and then departs; if yes, it asks, is your threshold $\geq 4$ ? If no, it takes on a value of 1 ; if yes, it takes on a value of 2 and leaves. Each aHPC only responds once to a reporter object. Given the cumulative fraction of dosed reporters having values 0,1 , and 2 , we can estimate the relative numbers of PP, MZ, and PC aHPCs in that Culture.

It is feasible to obtain analogous wet-laboratory information, e.g., by measuring any of a variety of macromolecules that exhibit zonation (Planas-Paz et al., 2016; Katoonizadeh, 2017). In general, results from wet-laboratory measurements that provide information about the heterogeneity of relevant mechanism component parts, component operations, and their organization would be among the multiattribute similarities requested on the right-hand side in Fig. 1D. However, there are no wet-laboratory measurements that will recover lost lobular structural and organizational properties that influence the phenomena of interest in vivo. Lobular architecture and intra-aHPC Mechanisms are entangled within Lobules and cannot be separated. Having achieved multiattribute similarities between Mouse Analogs and wetlaboratory measurements for multiple compounds (Yan et al., 2008a,b; Park et al., 2009, 2010) (left-hand side, Fig. 1), we use the ability to transition smoothly between Culture and Mouse Analogs (top, Fig. 1D) to simulate that entanglement.

Once we establish quantitative multiattribute similarities between Culture Analog and wet-laboratory counterparts, we can reconfigure Culture Analog aHPCs into an organized lobule structure within the Mouse Analog. The intra-aHPC model mechanisms will be the same. Virtual experiments on the resulting virtual Mouse Analogs can provide plausible Model Mechanism-based predictions of corresponding experiments in mice. The credibility of these predictions will be wholly dependent on the credibility of the Model Mechanisms and multiattribute similarities established on the right- and left-hand sides in Fig. 1D. The virtual Culture-to-Mouse translation can stand as a concrete, scientifically challengeable hypothesis explaining the in vivo-to-in vitro disconnect. The resulting Model Mechanism-based theory would be expected to provide the framework to develop more reliable interpretations of in vitro observations, which then may be used to improve extrapolations.

\section{Acknowledgments}

The UCSF BioSystems group supported this work. We thank Ryan Kennedy for constructive criticism.

\section{Authorship Contributions}

Participated in research design: Smith, Ropella, Hunt.

Conducted experiments: Smith, $\mathrm{Xu}$.

Contributed new reagents or analytic tools: Smith, Xu, Ropella. Performed data analysis: Smith, Xu.

Wrote or contributed to the writing of the manuscript: Smith, Hunt, Ropella.

\section{References}

Anundi I, Lähteenmäki T, Rundgren M, Moldeus P, and Lindros KO (1993) Zonation of acetaminophen metabolism and cytochrome P450 2E1-mediated toxicity studied in isolated periportal and perivenous hepatocytes. Biochem Pharmacol 45: 1251-1259.

Bowman CM and Benet LZ (2016) Hepatic clearance predictions from in vitro-in vivo extrapolation and the biopharmaceutics drug disposition classification system. Drug Metab Dispos 44:1731-1735.

Darden L (2008) Thinking again about biological mechanisms. Philos Sci 75:958-969.

Fraczek J, Bolleyn J, Vanhaecke T, Rogiers V, and Vinken M (2013) Primary hepatocyte cultures for pharmaco-toxicological studies: at the busy crossroad of various anti-dedifferentiation strategies. Arch Toxicol 87:577-610.

Godoy P, Hewitt NJ, Albrecht U, Andersen ME, Ansari N, Bhattacharya S, Bode JG, Bolleyn J, Borner C, Böttger J, et al. (2013) Recent advances in 2D and 3D in vitro systems using primary hepatocytes, alternative hepatocyte sources and nonparenchymal liver cells and their use in investigating mechanisms of hepatotoxicity, cell signaling and ADME. Arch Toxicol 87:1315-1530.

Hinson JA, Roberts DW, and James LP (2010) Mechanisms of acetaminopheninduced liver necrosis, in Adverse Drug Reactions (Uetrecht J ed) (Handbook of Experimental Pharmacology), vol 196, pp 369-405, Springer, Berlin.

Hunt CA, Ropella GE, Lam Tn, and Gewitz AD (2011) Relational grounding facilitates development of scientifically useful multiscale models. Theor Biol Med Model 8:35-66.

Katoonizadeh A (2017) Liver regeneration, in Liver Pathophysiology, pp 113-123, Elsevier, Amsterdam, Netherlands.

Kera Y, Penttilä KE, and Lindros KO (1988) Glutathione replenishment capacity is lower in isolated perivenous than in periportal hepatocytes. Biochem $J$ 254: 411-417.

Kirschner DE, Hunt CA, Marino S, Fallahi-Sichani M, and Linderman JJ (2014) Tuneable resolution as a systems biology approach for multi-scale, multicompartment computational models. Wiley Interdiscip Rev Syst Biol Med 6: 289-309.

LeCluyse EL, Witek RP, Andersen ME, and Powers MJ (2012) Organotypic liver culture models: meeting current challenges in toxicity testing. Crit Rev Toxicol $\mathbf{4 2}$ : 501-548.

Luke S, Cioffi-Revilla C, Panait L, Sullivan K, and Balan G (2005) MASON: a multiagent simulation environment. Simulation 81:517-527.

Mitchell JR, Jollow DJ, Potter WZ, Davis DC, Gillette JR, and Brodie BB (1973) Acetaminophen-induced hepatic necrosis. I. Role of drug metabolism. J Pharm Exp Therap. 187:185-194.

Ni HM, Williams JA, Jaeschke H, and Ding WX (2013) Zonated induction of autophagy and mitochondrial spheroids limits acetaminophen-induced necrosis in the liver. Redox Boil. 31:427-432.

Park S, Kim SH, Ropella GEP, Roberts MS, and Hunt CA (2010) Tracing multiscale mechanisms of drug disposition in normal and diseased livers. J Pharmacol Exp Ther 334:124-136.

Park S, Ropella GEP, Kim SH, Roberts MS, and Hunt CA (2009) Computational strategies unravel and trace how liver disease changes hepatic drug disposition. $J$ Pharmacol Exp Ther 328:294-305.

Petersen BK and Hunt CA (2016) Developing a vision for executing scientifically useful virtual biomedical experiments, in Proceedings of the 2016 Spring Simulation Multiconference. pp 697-706, Society for Computer Simulation International, San Diego, CA

Petersen BK, Ropella GE, and Hunt CA (2014) Toward modular biological models: defining analog modules based on referent physiological mechanisms. BMC Syst Biol 8:95-112.

Petersen BK, Ropella GE, and Hunt CA (2016) Virtual experiments enable exploring and challenging explanatory mechanisms of immune-mediated P450 down-regulation. PLoS One 11:e0155855.

Planas-Paz L, Orsini V, Boulter L, Calabrese D, Pikiolek M, Nigsch F, Xie Y, Roma G, Donovan A, Marti P, et al. (2016) The RSPO-LGR4/5-ZNRF3/RNF43 module controls liver zonation and size. Nat Cell Biol 18:467-479. 
Pogson M, Smallwood R, Qwarnstrom E, and Holcombe M (2006) Formal agent-based modelling of intracellular chemical interactions. Biosystems 85:37-45.

Poulin P (2016) The need for human exposure projection in the interpretation of preclinical in vitro and in vivo ADME tox data, in Drug Discovery Toxicology: From Target Assessment to Translational Biomarkers (Will Y, McDuffie JE, Olaharski AJ, and Jeffy BD eds) pp 67-81, John Wiley \& Sons, Inc, Hoboken, NJ.

Roberts DW, Bucci TJ, Benson RW, Warbritton AR, McRae TA, Pumford NR, and Hinson JA (1991) Immunohistochemical localization and quantification of the 3 -(cystein-S-yl)-acetaminophen protein adduct in acetaminophen hepatotoxicity. Am J Pathol 138:359-371.

Ropella GEP and Hunt CA (2010) Cloud computing and validation of expandable in silico livers. BMC Syst Biol 4:168.

Saito C, Lemasters JJ, and Jaeschke H (2010) c-Jun N-terminal kinase modulates oxidant stress and peroxynitrite formation independent of inducible nitric oxide synthase in acetaminophen hepatotoxicity. Toxicol Appl Pharmacol 246:8-17.

Sastre J, Rodriguez JV, Pallardó FV, Gasco E, Asensi M, Ferrer JV, Miquel J, and Viña $J$ (1992) Effect of aging on metabolic zonation in rat liver: acinar distribution of GSH metabolism. Mech Ageing Dev 62:181-190.

Smith AK, Petersen BK, Ropella GE, Kennedy RC, Kaplowitz N, Ookhtens M, and Hunt CA (2016) Competing mechanistic hypotheses of acetaminopheninduced hepatotoxicity challenged by virtual experiments. PLOS Comput Biol 12:e1005253.

Smith AK, Ropella GE, Kaplowitz N, Ookhtens M, and Hunt CA (2014) Mechanistic agent-based damage and repair models as hypotheses for patterns of necrosis caused by drug induced liver injury, in Proceedings of the 2014 Summer Computer Simulation Multiconference. pp 112-120, Society for Computer Simulation International, San Diego, CA.

Soldatow VY, Lecluyse EL, Griffith LG, and Rusyn I (2013) In vitro models for liver toxicity testing. Toxicol Res (Camb) 2:23-39.

Tetsuka K, Ohbuchi M, and Tabata K (2017) Recent progress in hepatocyte culture models and their application to the assessment of drug metabolism, transport, and toxicity in drug discovery: the value of tissue engineering for the successful development of a microphysiological system. J Pharm Sci 106:2302-2311.

Vellonen KS, Malinen M, Mannermaa E, Subrizi A, Toropainen E, Lou YR, Kidron H, Yliperttula M, and Urtti A (2014) A critical assessment of in vitro tissue models for ADME and drug delivery. $J$ Control Release 190:94-114.

Yan L, Ropella GE, Park S, Roberts MS, and Hunt CA (2008a) Modeling and simulation of hepatic drug disposition using a physiologically based, multi-agent in silico liver. Pharm Res 25:1023-1036.

Yan L, Sheihk-Bahaei S, Park S, Ropella GE, and Hunt CA (2008b) Predictions of hepatic disposition properties using a mechanistically realistic, physiologically based model. Drug Metab Dispos 36:759-768.

Address correspondence to: C. Anthony Hunt, Bioengineering and Therapeutic Sciences, 513 Parnassus Ave., HSE-1119, University of California, San Francisco, CA 94143-0912. E-mail: a.hunt@ucsf.edu 